\title{
НЕЙРОСЕТЕВОЙ ПОДХОД ОПРЕДЕЛЕНИЯ ПОКАЗАТЕЛЕЙ ЛЯПУПОВА ДЛЯ ХАОТИЧЕСКИХ ПРОЦЕССОВ
}

\author{
В. Головко, Ю. Савицкий
}

Брестский государственный технический университет

224017, г.Брест, ул.Московская, 267

В работе рассматриваются новые методы и алгоритмы идентификаиии хаотических проиессов, основанные на моделях многослойных нейронных сетей. Предложен нейросетевой алгоритм вычисления наибольшего показателя Ляпунова для различного рода хаотических временных рядов. Рассмотрена оригинальная методика вычисления спектра показателей Ляпунова, которая базируется на использовании нелинейной многослойной нейронной сети и метода ортогонализачии Грамма-Шмидта. Предложенные методы характеризуются малым объемом исходных данных и высокой скоростью вычислений по сравнению с традиционными. Экспериментальные исследов ания методов на основе временных последовательностей Энона и Лоренца демонстрируют высокое соответствие полученных результатов эталонным значениям.

Ключевые слова: хаотический процесс, многослойная нейронная сеть, наибольший показатель Ляпунова, спектр Ляпунова.

\section{ВВЕДЕНИЕ}

Хаотическое поведение системы характеризуется странными аттракторами, которые имеют фрактальную размерность и обладают свойством масштабной инвариантности [1].

Обработку хаотических процессов можно разделить на ряд этапов. Первый этап - это анализ временного ряда. В результате его выполнения определяются размерность пространства вложения и временная задержка сигнала [2,3]. Определение параметров вложения обеспечивает максимальную предсказуемость временного ряда и может быть использована для выбора оптимального размера окна (количество входных элементов) в прогноз ирующей нейронной сети. Используя результаты предыдущего этапа можно осуществить идентификацию хаотического процесса путем вычисления максимального показателя Ляпунова, а также осуществить прогнозирование и фазовую реконструкцию хаотиче ского процесса. Основной проблемой здесь является обработка хаотических проце ссов по наблюдаемой реализации. В этом случае при малом объеме исходных данных проблематично использовать стандартные аналитические подходы как для реконструкции аттрактора, так и для вычисления спектра Ляпунова.
Поэтому альтернативой здесь является разработка нейросетевого подхода для обработки хаотических процессов, что, как будет показано ниже, позволяет значительно снизить трудоемкость вычислений.

В данной статье описывается нейросетевой подход для определения наибольшего показателя Ляпунова и спектра Ляпунова хаотических процессов.

\section{1. ОПРЕДЕЛЕНИЕ НАИБОЛЬШЕГО ПОКАЗАТЕЛЯ ЛЯПУНОВА}

Критерием хаотичности системы является наличие положительного наибольшего показателя Ляпунова [4]. Поэтому определение такого показателя позволяет идентифицировать динамическую систему с точки зрения присутствия в ней хаотического поведения. Показатель Ляпунова характеризует степень экспоненциального разбегания траекторий. Пусть $d(0)$ является начальным расстоянием между двумя точками, $d(n)$ - расстояние между этими точками через $n$ шагов. Тогда наибольший показатель Ляпунова определяется следующим соотношением

$$
\lambda=\lim _{n \rightarrow \infty} \frac{1}{n} \ln \frac{d(n)}{d(0)}
$$

Для вычисления старшего показателя 
Ляпунова применяется следующий метод [5]. Пусть известна одна координата хаотического процесса $X(t), \quad t=\overline{1, p}$. Тогда вначале необходимо определить размерность пространства вложения $m$ и временную задержку $\phi[7,8]$. В результате получим следующий набор точек:

$$
\begin{aligned}
& X(t)=(x(t), x(t+\phi), \ldots, x(t+(m-1) \phi)), \\
& t=\overline{1, p} .
\end{aligned}
$$

Выбираем исходную точку $X(0)$ в области притяжения аттрактора и находим ближайшую к ней точку $X_{l}(0)$ таким образом, чтобы расстояние между ними было достаточно малым:

$$
d(0)=|X(0)-X \tau(0)| \mathrm{H}^{\prime \prime} 10^{-8}
$$

Затем отслеживаем эволюцию этих двух точек на фазовой траектории до тех пор, пока $d(n)<1$

Строим график зависимости $\ln d(n)$ от $n$ и прямую регрессии, наклон которой соответствует наибольшему показателю Ляпунова. Такой алгоритм базируется на эргодической теореме В.И. Оселедеца $[5,6]$ которая утверждает, что экспоненциальное расхождение двух случайно выбранных точек на аттракторе с единичной вероятностью характеризует старший показатель Ляпунова. Приведённый выше метод характеризуется большой вычислительной сложностью и невозможностью применения для малого объёма исходных данных. Это связано с тем, что трудно найти две точки ряда, отстоящие друг от друга на расстояния меньше чем $10^{-8}$. Особенно это проблематично для реальных данных. Одним из путей для преодоления этого недостатка является применение нейронных сетей для вычисления старшего показателя Ляпунова. Ключевой идеей данного метода является вычисление при помощи прогнозирующей нейронной сети расхождения двух близлежащих траекторий на $n$ шагов вперёд.

Нейронная сеть будет состоять из $k=m-1$ входных нейронов, где $m-$ размерность пространства вложения, $p$ скрытых и одного выходного нейронного элемента (рис. 1).

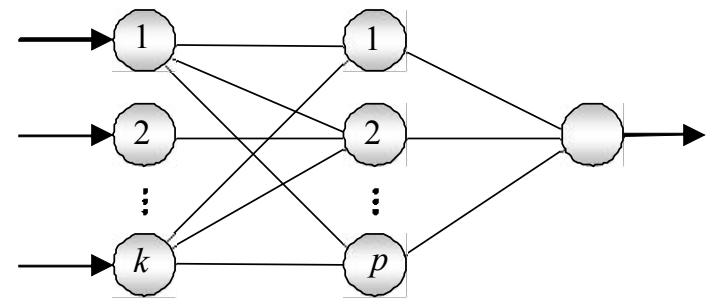

Рис. 1. Прогнозирующая нейронная сеть
Вначале необходимо обучить такую нейронную сеть прогнозированию в соответствии с методом скользящего окна:

$$
x(t+i \phi)=F(x(t+(i-1) \phi), x(t+(i-2) \phi), \ldots,
$$$$
x(t+(i-k) \phi)), i=\overline{1, n} \text {. }
$$

После обучения сети легко осуществить эволюцию двух точек на фазовой траектории, используя итерационный подход.

Таким образом, ключевой идеей предлагаемого метода $[7,8]$ является вычисление при помощи прогнозирующей нейронной сети расхождения двух близлежащих траекторий на $n$ шагов вперёд, используя итерационный подход. Эта процедура может быть представлена следующим алгоритмом:

1. Обучаем нейронную сеть на прогнозирование по методу скользящего окна.

2. Выбираем любую точку $x(t)$ из обучающей выборки и формируем следующий набор данных: $\{x(t), x(t-\phi), \ldots, x(t-(k-1) \phi)\}$, где $k-$ размер окна.

3. Вычисляем $\{x(t+\phi), x(t+2 \phi), \ldots, x(t+n \phi)\}$ используя многошаговый прогноз

4. $x(t+i \phi)=F(x(t+(i-1) \phi), x(t+(i-2) \phi), \ldots$, $x(t+(i-k) \phi))$, где $i=\overline{1, n}, F-$ нелинейная функция.

5. Вычисляем $x^{\prime}(t)=x(t)+d_{0}$, где $d_{0} \approx 10^{-8}$ и подавая на сеть $\left\{x^{\prime}(t), x(t-\phi), \ldots\right.$, $x(t-(k-1) \phi)\}$ повторяем шаг 3 для получения $x^{\prime}(t+i \tau), i=\overline{1, n}$

6. Оцениваем

$\ln d_{i}=\ln \left|x^{\prime}(t+i \tau)-x(t+i \tau)\right|$

$i=\overline{1, n}$ и выбираем только точки, где $\ln d<0$.

7. Строим график $\ln \left(d_{n}\right)$ от $n$.

8. Строим прямую регрессии для выбранных точек и вычисляем её наклон, который равен наибольшему показателю Ляпунова.

Данный метод для вычисления 1 был опробирован на многослойном персептроне. Для эксперимента использовалась нейронная сеть с 7 входными элементами, 5 скрытыми и 1 выходным нейроном для предсказания временных рядов Лоренца и Энона. Элементы скрытого слоя имели сигмоидную функцию активации, а выходной элемент - линейную. Для обучения сети использовался алгоритм 
обратного распространения ошибки с адаптивным шагом. Обучающая выборка состояла из 70 элементов для данных Энона и 100 элементов для данных Лоренца. Рис. 2 показывает график $\ln d(n)$ от $n$ и прямую регрессии, которая определяет показатель Ляпунова. Оцененное значение $€=0.43$ близко к ожидаемому значению 0.419 для данных Энона. Прямая регрессии и функция $\ln \left(d_{n}\right)$ от $n$ для процесса Лоренца представлены на рис. 3. Наибольший показатель Ляпунова равен 0.98 (ожидаемое значение 0.906). Можно заметить, что нейронная сеть осуществляет довольно точную оценку показателя Ляпунова.

Как видно, очевидное преимущество предложенного подхода в сравнении с традиционным - простота и точность.

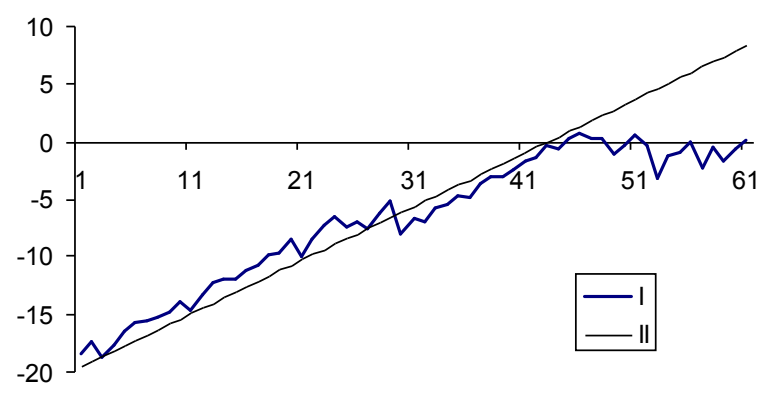

Рис. 2. I - Изменение расстояния между
двумя близкими траекториями для
аттракт ора Энона, II - прямая регрессии

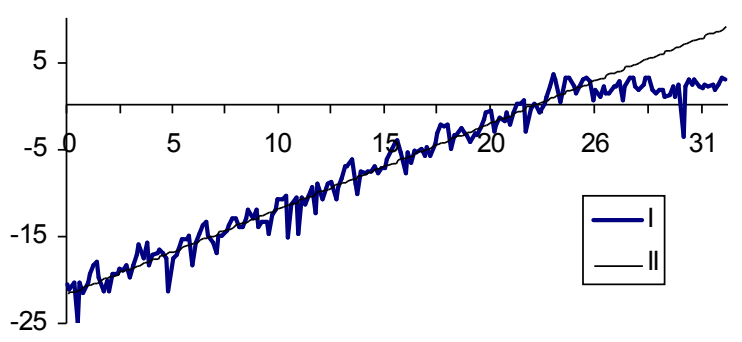

\section{двумя близкими траекториями для аттрактора Лоренца, II - прямая регрессии}

\section{2. ОПРЕДЕЛЕНИЕ СПЕКТРА ЛЯПУНОВА}

Пусть дана динамическая система, описываемая $n$ дифференциальными уравнениями. Такая система имеет $n$ показателей Ляпунова $л_{i}(i=1,2, \ldots, n)$, которые называются спектром Ляпунова. Спектр Ляпунова характеризует чувствительность динамической системы к начальным условиям. Наибольший показатель Ляпунова определяет в среднем экспоненциальную скорость разбегания двух траекторий, исходящих из близких точек. Для хаотического поведения наибольший показатель Ляпунова является положительным. Проведённые выше рассуждения можно обобщить на спектр Ляпунова, рассматривая в качестве начальных условий небольшую $n$ мерную сферу. С течением времени такая сфера деформируется в эллипсоид, имеющий $n$ главных полуосей. Спектр Ляпунова характеризует изменение длин главных полуосей эллипсоида с течением времени. Пусть, например, показатели Ляпунова расположены в порядке убывания

$$
\lambda_{1} \geq \lambda_{2} \geq \ldots \lambda_{n}
$$

При этом $\lambda_{1}$ соответствует большей оси эллипсоида, $\lambda_{2}-$ следующий по величине оси и т.д. Тогда $i$-й показатель Ляпунова определяется следующим образом

$$
\lambda_{i}=\lim _{t \rightarrow \infty} \frac{1}{t} \ln \frac{l_{i}(t)}{l_{i}(0)}
$$

где $l_{i}(0)$ и $l_{i}(t)-$ соответственно длины $i$-ой оси эллипсоиды в начальный момент времени и момент времени $t$. Таким образом, каждый показатель Ляпунова характеризует изменение размеров эллипсоида по одной из его главных осей. Для их вычисления необходимо отслеживать изменение длин соответствующих векторов вдоль фазовой траектории. Чтобы это осуществить используют в основном численный подход, основанный на выводе уравнений динамики системы в вариациях [9]. В процессе вычислений производится процедура ортогонализации Грамма-Шмидта. Однако такой метод требует наличия динамических уравнений системы и не подходит при обработке экспериментальных данных. Основная проблема при вычислении показателей Ляпунова состоит в трудности отслеживания эволюции расстояния между точками фазовой траектории с течением времени. Поэтому разработка эффективного и робастного метода определения спектра Ляпунова является важной задачей [10]. Для её решения можно предложить простой метод, основанный на применении нейронной сети для вычисления расстояний между точками фазовой траектории с течением времени. Пусть состояние динамической системы в каждый момент времени задаётся $n$-мерным вектором

$$
\mathbf{X}(\mathrm{t})=\left(\mathrm{X}_{1}(\mathrm{t}), \mathrm{X}_{2}(\mathrm{t}), \ldots, \mathrm{X}_{\mathrm{n}}(\mathrm{t})\right)
$$


Предположим, что известны временные зависимости $X_{i}(t) \quad$ соответствующих переменных на определённом интервале времени.

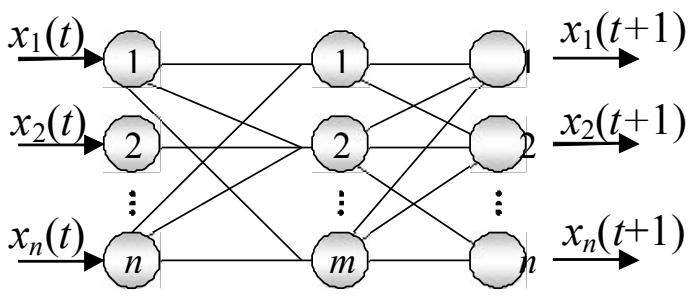

Рис. 4. Прогнозирующая нейронная сеть

Тогда сгенерируем нейронную сеть, которая на основе предыдущего состояния динамической системы прогнозирует следующее состояние. Она будет состоять из $n$ входных, $m$ скрытых и $n$ выходных нейронных элементов (рис. 4). Выходные значения нейронной сети определяются следующим образом:

$$
X(t+1)=F(X(t))
$$

После обучения такой сети, можно определить как состояние динамической системы в произвольный момент времени, так и эволюцию точек фазовой траектории, используя только наблюдаемые реализации. В процессе вычислений необходимо проводить процедуру ортогонализации Грамма-Шмидта. Пусть $\left|w_{i}(t)\right|$ - длина $i$-го вектора в момент времени $t$. Она характеризует размер вектора вдоль $i$-ой оси эллипсоида. Тогда $i$-й показатель Ляпунова можно определить следующим образом:

$$
\lambda_{i}=\lim _{p \rightarrow \infty} \frac{1}{p} \sum_{t=1}^{p} \ln \frac{\left|W_{i}(t)\right|}{\left|W_{i}(t-1)\right|}
$$

Определяя соответствующие длины векторов $\left|W_{i}(t)\right|$ при помощи нейронной сети, можно вычислить спектр Ляпунова.

Рассмотрим алгоритм вычисления показателей Ляпунова. Он состоит из следующих шагов:

1. Выбираем начальную точку из области притяжения аттрактора, координаты которой равняются:

$$
N(0)=\left(x_{1}(0), x_{2}(0), \ldots, x_{n}(0)\right)
$$

2. Задаем достаточно малое число е $=10^{-8}$ и определяем координаты следующих $n$ точек в соответствии с правилом:

$$
\begin{aligned}
& A_{1}(0)=\left(x_{1}(0), x_{2}(0), \ldots, x_{n}(0)\right) \\
& A_{2}(0)=\left(x_{1}(0), x_{2}(0)+\varepsilon, \ldots, x_{n}(0)\right) \\
& \ldots \ldots \ldots \ldots \ldots \ldots \ldots \ldots \ldots \ldots \ldots \ldots \ldots \\
& A_{n}(0)=\left(x_{1}(0), x_{2}(0), \ldots, x_{n}(0)+\varepsilon\right)
\end{aligned}
$$

В результате выполнения этого пункта получаем следующий набор ортогональных векторов:

$$
\begin{gathered}
N A_{1}(0)=(\varepsilon, 0, \ldots, 0) \\
N A_{2}(0)=(0, \varepsilon, \ldots, 0) \\
\ldots \ldots \ldots \ldots \ldots \\
N A_{n}(0)=(0,0, \ldots, \varepsilon)
\end{gathered}
$$

3. Определяем длину каждого вектора:

$$
\left|N A_{i}(0)\right|=\left|W_{i}(0)\right|=\varepsilon
$$

где $i=\overline{1, n}$

4. Последовательно подаем на входы нейронной сети координаты точек $N(0), \mathrm{A}_{1}(0)$, $\mathrm{A}_{2}(0), \ldots, \mathrm{A}_{n}(0)$ в момент времени $t=0$. В результате получаем на выходах нейронной сети координаты точек в следующий момент времени $t=t+1$ :

$$
\begin{aligned}
& N(0)=\left(x_{1}(1, N), x_{2}(1, N), \ldots, x_{n}(1, N)\right) \\
& A_{1}(0)=\left(x_{1}\left(1, A_{1}\right), x_{2}\left(1, A_{1}\right), \ldots, x_{n}\left(1, A_{1}\right)\right) \\
& A_{2}(0)=\left(x_{1}\left(1, A_{2}\right), x_{2}\left(1, A_{2}\right), \ldots x_{n}\left(1, A_{2}\right)\right) \\
& \ldots \ldots \ldots \ldots \ldots \ldots \ldots \ldots \ldots \ldots \ldots \ldots \\
& A_{n}(0)=\left(x_{1}\left(1, A_{n}\right), x_{2}\left(1, A_{n}\right), \ldots, x_{n}\left(1, A_{n}\right)\right)
\end{aligned}
$$

(9)

где $x_{i}(1, A)-i$-я координата точки $A_{j}$ в момент времени $t=1$.

В результате выполнения этого пункта получаем следующий набор неортогональных векторов:

$$
\begin{aligned}
& N A_{1}(1)=W_{1}(1)=\left(w_{11}, w_{21}, \ldots, w_{n 1}\right) \\
& N A_{2}(1)=W_{2}(1)=\left(w_{12}, w_{22}, \ldots, w_{n 2}\right) \\
& \ldots \ldots \ldots \ldots \ldots \ldots \ldots \ldots \ldots \ldots \ldots \ldots \ldots \\
& N A_{n}(1)=W_{n}(1)=\left(w_{1 n}, w_{2 n}, \ldots, w_{n n}\right)
\end{aligned}
$$

Здесь $w_{i j}-i$-ая координата $j$-го вектора. Она определяется следующим образом: 


$$
w_{i j}=x_{i}\left(1, A_{j}\right)-x_{i}(1, N)
$$

5. В соответствии с процедурой ГраммаШмидта производим преобразование базиса $\left(W_{1}(1), W_{2}(1), \ldots, W_{n}(1)\right)$ в ортонормированный базис. Для этого выполняем следующие действия:

a) В качестве первого вектора ортонормированного базиса выберем

$$
W_{1}^{\prime}(1)=\left(\frac{w_{11}}{\left|W_{1}(1)\right|}, \frac{w_{21}}{\left|W_{1}(1)\right|}, \ldots, \frac{w_{n 1}}{\left|W_{1}(1)\right|}\right)
$$

где

$$
\left|W_{1}(1)\right|=\sqrt{w_{11}^{2}+w_{21}^{2}+. .+w_{n 1}^{2}}
$$

б) Остальные векторы определяются рекурсивным способом в соответствии со следующими выражениями

$$
\begin{aligned}
& W_{i}(1)=W_{i}(1)-\sum_{j=1}^{p}\left(W_{i}^{T}(1) W_{j}^{\prime}(1)\right) W_{j}^{\prime}(1), \\
& \left|W_{i}(1)\right|=\sqrt{w_{1 i}^{2}+w_{2 i}^{2}+. .+w_{n i}^{2}},(15) \\
& W_{i}^{\prime}(1)=\left(\frac{w_{1 i}}{\left|W_{i}(1)\right|}, \frac{w_{2 i}}{\mid W_{i}(1)}, \ldots, \frac{w_{n i}}{\left|W_{i}(1)\right|}\right)
\end{aligned}
$$

где $i=\overline{2, n}$

6. Для каждого полученного вектора находим логарифмы отношения длин векторов в два последовательных момента времени:

$$
S_{i}(1)=\ln \frac{\left|W_{i}(1)\right|}{\left|W_{i}(0)\right|},
$$

где $i=\overline{1, n}$.

7. Формируем новый набор точек, координаты которых определяются следующим образом:

$$
\begin{aligned}
& N(1)=\left(x_{1}(1, N), x_{2}(1, N), \ldots, x_{n}(1, N)\right) \\
& A_{1}(1)=\left(\bar{x}_{1}\left(1, A_{1}\right), \bar{x}_{2}\left(1, A_{1}\right), \ldots, \bar{x}_{n}\left(1, A_{1}\right)\right) \\
& A_{2}(1)=\left(\bar{x}_{1}\left(1, A_{2}\right), \bar{x}_{2}\left(1, A_{2}\right), \ldots, \bar{x}_{n}\left(1, A_{2}\right)\right) \\
& \ldots \ldots \ldots \ldots \ldots \ldots \ldots \ldots \ldots \ldots \ldots \ldots . \ldots \ldots \ldots \ldots \\
& A_{n}(1)=\left(\bar{x}_{1}\left(1, A_{n}\right), \bar{x}_{2}\left(1, A_{n}\right), \ldots, \bar{x}_{n}\left(1, A_{n}\right)\right) \\
& \text { где } \bar{x}_{i}\left(1, A_{j}\right)=w_{i j}+x_{i}(1, N) .
\end{aligned}
$$

8. Продолжаем процедуру, начиная с пункта
3 для $t=\overline{1, p}$, где $p \mathrm{H}^{\prime \prime} 1000$

9. Определяем спектр Ляпунова в соответствии со следующим выражением:

$$
\lambda_{i}=\frac{1}{p} \sum_{t=1}^{p} S_{i}(t),
$$

где $i=\overline{1, n}$

Определяемые при этом показатели Ляпунова упорядочены по убыванию

$$
\lambda_{1}>\lambda_{2}>\ldots>\lambda_{n}
$$

На рисунках 5-7 изображены экспериментальные исследования по определению спектра Ляпунова рассмотренным выше методом для хаотических процессов Энона и Лоренца. Как видно из рисунков для процесса Энона были получены следующие показатели Ляпунова: 0.442 и -1.625 , что хорошо согласуется с эталонными значениями 0.418 и -1.622 . Соответственно для процесса Лоренца спектр Ляпунова составил $0.777,0.003$ и -14.472, что также достаточно точно соответствует эталонным значениям $0.906,0,-$ 14.472. Таким образом, разработанный в данной работе нейросетевой методпозволяет определить спектр Ляпунова основываясь только на наблюдаемых реализациях.

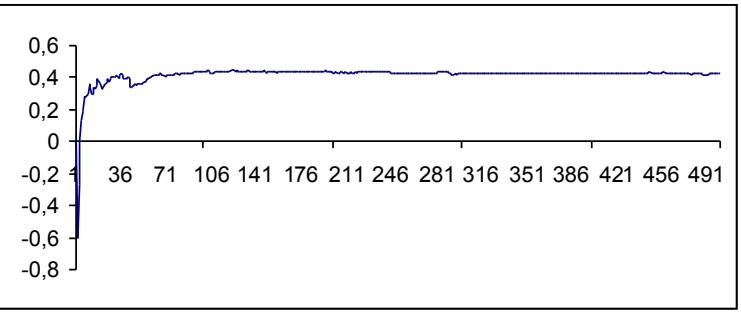

Рис. 5. Вычисление первого показателя Ляпунова для рядов Энона

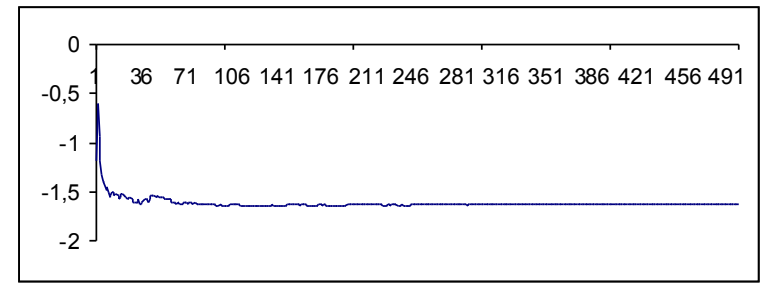

Рис. 6. Вычисление второго показателя Ляпунова для рядов Энона

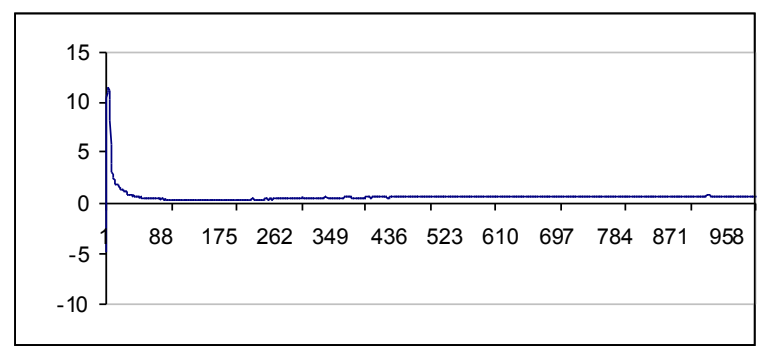



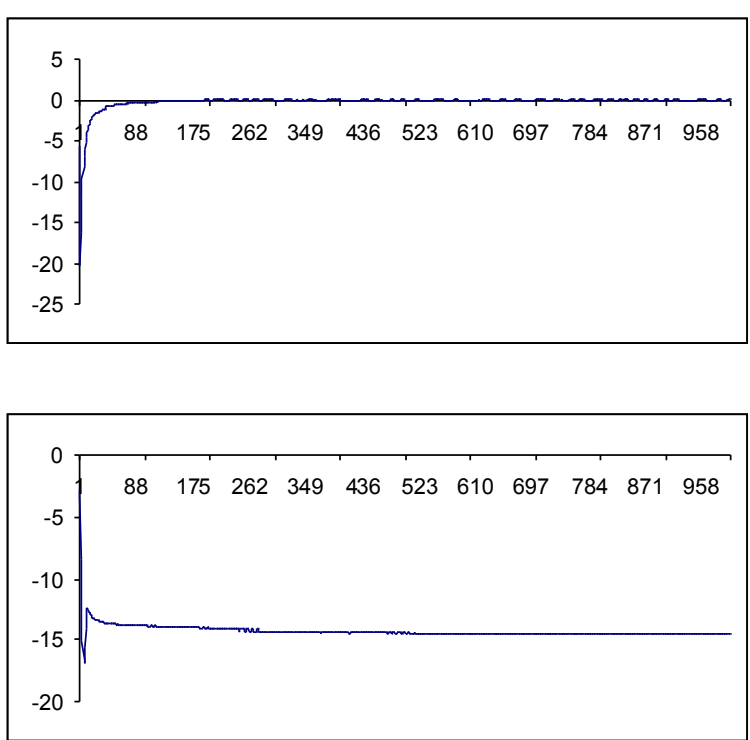

Рис. 7. Вычисление показателей Ляпунова для рядов Лоренца

\section{ЗАКЛЮЧЕНИЕ}

В работе рассмотрены методы и алгоритмы определения показателей Ляпунова, являющихся чрезвычайно важными параметрами идентификации хаотического поведения различного рода динамических процессов. Разработан нейросетевой метод для вычисления наибольшего показателя Ляпунова, который позволяет вычислять искомый показатель при малом объёме исходной временной последовательности, и обладает меньше вычислительной сложностью по сравнению с традиционными методами. Предложен нейросетевой метод для определения спектра Ляпунова, достоинствами которого являются возможность определения показателей Ляпунова на основе наблюдаемых реализаций хаотического процесса. Важной практической направленностью методов является возможность анализа хаотическихпроцессов, представленных небольшими объемами их реализаций.

\section{СПИСОК ЛИТЕРАТУРЫ}

[1] H. Schuster. Deterministic chaos. An introduction. Physic-Verlag, Weinhheim, 1984, p.240.

[2] A. Fraser, H. Swinney. Independent coordinates for strange attractors from mutual information. Phys. Rev. A 33, 1134 (1986).

[3] M. Kennel, R. Brown, H. Abarbanel. Determining embedding dimension for phase-space reconstruction using a geometrical construction. Phys. Rev. A 45, 3403 (1992).

[4] H. Kantz. A robust method to estimate the maximal Lyapunov exponent of a time series. Phys. Lett. A 185, 77 (1994).
[5] G. Benettin, L. Galgani, J.-M. Strelcyn, Kolmogorov entropy and numerical experiments, Physical Review A 14, 1976, pp. 2338-2345.

[6] Оселедеи В.И. Мультипликативная эргодическая теорема. Характеристические показатели Ляпунов а динамических систем // Тр. Моск. Мат. Обществ а, 1968, T.19, с. 179220.

[7] V. Golovko, Y. Savitsky, N. Maniakov. Modeling Nonlinear Dynamic Using Multilayer Neural Networks. Proceedings of the Workshop Intelligent Data Acquisition and Advanced Computing Systems: Technology and Applications (IDAACS'2001), Foros, Ukraine, July 1-4 2001, PP. 197-202.

[8] V. Golovko, Y. Savitsky, N. Maniakov, V.Rubanov, Some Aspects of Chaotic Time Series Analysis, Proceedings of the 2nd International Conference on Neural Networks and Artificial Intelligence, October 2-5, 2001, Minsk, Belarus, pp. 66-69.

[9] G. Benettin, L. Galgani, A. Giorgilli, J.-M. Strelcyn, Lyapunov characteristic exponents for smooth dynamical systems and for Hamiltonian systems: A method for computing all of them. P. I: Theory. P. II: Numerical applications, Meccanica, Vol. 15, 1980, pp. 9-30.

[10] X. Zeng, R. Eykholt and R.A. Pielke, Estimating the Lyapunov-Exponent Spectrum from shot Time Series of Low Precision, Physical Review Letter 66, 1991, pp. 3229-3232.

Головко Владимир Адамович, кандидат технических наук, в 19842. закончил МВТУ им. Баумана, а в 19892. аспирантуру ИТК АНРБ. С 19892. по 19942. работал в Брестском отделении ИТК АН РБ. С 19942. - доцент кафредры "ЭВМ и Системы" БГТУ; с 20012. профрессор кафредры “ЭВМ и Системы” БГТУ. Имеет более 133 научных работ. В БГТУ Головко В.А. организовал группу исследователей, которые занимаются различными аспектами нейронных сетей. Имеет 6 аспирантов, которые активно работают в этом направлении. С 1999 г. Головко В. А. опубликованы 3 монографии по теоретическим и практическим аспектам нейронных систем. Головко В. А. является руководителем 3 международных проектов, председателем SIG INNS.

Савицкий Юрий Викторович, кандидат технических наук, в 1993е. закончил Брестский политехнический институт. Область научных интересов - интеллектуальные нейронные системы прогнозирования, нейросетевые методы анализа хаотических 
сигналов. В 2000 е. защитил кандидатскую диссертацию на тему «Методы и средства генерации искусственных нейронных сетей для решения задач прогнозирования». Имеет более 50 научных работ. С 20012. - доцент кафеедры “ЭВМ и Системы” БГТУ. 